\title{
L'intérêt d'une approche systémique des dynamiques de végétations en phytosociologie forestière tropicale : cas de la forêt classée de Sanaimbo à Bongouanou/Dimbokro (Côte d'Ivoire)
}

\author{
N. J. KASSI ${ }^{1,2^{*}}$, O.D. YONGO ${ }^{2,3}$, E. AKE ASSI ${ }^{1}$ et G. DECOCQ ${ }^{2}$ \\ ${ }^{1}$ Laboratoire de botanique, Université de Cocody-Abidjan (Côte d'Ivoire), 22 BP 582 Abidjan 22 ; UFR \\ Biosciences. \\ ${ }^{2}$ Université de Picardie Jules Verne, Unité de recherche « Dynamiques des Systèmes Anthropisés » (JE 2532 \\ DSA), 1 rue des Louvels, F-80037 Amiens Cedex (France). \\ ${ }^{3}$ Laboratoire de biodiversité végétale et fongique, Faculté des Sciences, Université de Bangui, BP 908 \\ (République Centrafricaine). \\ *Auteur correspondant, E-mail : kassindja@yahoo.fr
}

\section{RESUME}

Dans cet essai, les auteurs proposent d'appliquer aux forêts tropicales la démarche systémique utilisée en phytosociologie depuis 1984 en Europe. Après en avoir rappelé le principe et les concepts fondamentaux, une classification opérationnelle des transformations spatio-temporelles est présentée. Un exemple d'application aux forêts denses semi décidues (forêt classée de Sanaimbo, Côte d'Ivoire) est proposé à partir de données phytosociologiques synusiales. Les principaux apports en matière de phytosociologie forestière et de foresterie sont évoqués : sur un plan fondamental: compréhension des phénomènes phytodynamiques, interprétation des écotones, analyse du fonctionnement des écosystèmes, analyses fonctionnelles comparatives et structuralistes ; sur un plan appliqué : bioindication, évaluation des potentialités forestières ; génie phyto-écologique appliqué à la gestion forestière durable.

(C) 2011 International Formulae Group. All rights reserved.

Mots clés : Successions écologiques, Synusie, Systémique, Structuralisme.

\section{INTRODUCTION}

L'approche systémique qualitative des phénomènes phytosociologiques est une démarche originale qui a été introduite en phytosociologie par De Foucault en 1984 pour comprendre les multiples interactions existant entre les différents groupements végétaux des prairies hygrophiles (De Foucault, 1984). Elle a depuis été étendue à d'autres types de végétations, notamment arbustives (De Foucault, 1991) et aux forêts tropicales
(Senterre, 2005), confirmant ses nombreuses potentialités, tant sur un plan fondamental qu'appliqué.

Nous proposons ici d'étendre son champ d'application aux végétations forestières tropicales, afin de mettre en évidence les nombreux phénomènes phytodynamiques sur lesquels repose le fonctionnement des écosystèmes forestiers. Nous déclinons également la classification opérationnelle des transformations 
élémentaires proposée par Decocq (1997a), utilisable pour la description de ces phénomènes. Nous prendrons l'exemple d'une forêt dense semi décidue (forêt classée de Sanaimbo, Centre-Est de la Côte d'Ivoire), pour laquelle une typologie phytosociologique a été récemment effectuée (Kassi, 2006 ; Kassi et Decocq, 2007) pour concrétiser nos propos. Enfin, nous envisageons les principaux apports de cette approche originale en phytosociologie forestière tropicale et plus généralement en foresterie.

\section{Principe général et justification}

L'idée selon laquelle la forêt tropicale représentait un écosystème stable et immuable dans le temps est désormais révolue. Pour s'en persuader, il suffit d'observer les différents aspects que prend, par exemple, une forêt dense semi décidue à la saison sèche et à la saison des pluies, ou encore de comparer la végétation forestière ivoirienne actuelle aux descriptions plus anciennes. Même en dehors de toute intervention humaine, il existe une véritable dynamique interne de la végétation forestière, se manifestant sous la forme d'une succession de communautés végétales au cours du temps (Hartshorn, 1980). Cette succession se traduit par des modifications texturales (composition floristique) et structurelles (architecture) du tapis végétal, soit spontanées, soit induites par les modifications des conditions écologiques environnantes. L'appréhension de ces phénomènes phytodynamiques est compliquée par le fait que tous les ensembles structurels de la végétation forestière tropicale ne sont pas obligatoirement touchés (cas des forêts denses semis décidues; la strate sous arbustive ne perdant pas son feuillage à la mauvaise saison), et lorsqu'ils le sont, pas toujours avec la même intensité et/ou de manière synchrone. Dans le cas de successions spontanées, le dynamisme est étroitement lié à l'éthologie et au pouvoir concurrentiel de ces espèces, alors que dans le cas de successions induites, il est corrélé avec la sensibilité et le pouvoir de résolution des espèces vis-à-vis des conditions du milieu.
Même lorsqu'on est en présence d'une forêt tropicale climacique, il existe une dynamique interne de la végétation forestière, qui assure l'autonomie «l'équilibre » de l'écosystème, et qui est à la base des cycles sylvigénétiques spontanés.

La structure temporelle de la végétation constitue une limite importante à la méthodologie phytosociologique. Celle-ci présente en effet l'inconvénient de donner une image «figée » de la végétation, ce qui ne correspond pas à la réalité temporelle du phénomène observé. L'étude phytosociologique d'une formation végétale se résume finalement à la réalisation de «clichés photographiques » des individus d'association végétale les plus typiques, en évitant fréquemment les groupements intermédiaires (les «zones de contact»), ce qui revient en quelque sorte à idéaliser l'image d'association végétale (Decocq, 1997a). C'est d'ailleurs ce qui l'oppose fondamentalement à la théorie du continuum des anglo-saxons. Selon Decocq (1997b), la phytosociologie ne peut plus se permettre de continuer d'aller plus avant dans l'évolution de ses concepts sans surmonter ce handicap de l'idéalisation et sans prendre en considération la véritable dimension temporelle de son objet d'étude. L'approche systémique élaborée par De Foucault (1984) fournit les moyens d'y parvenir, dans la mesure où elle donne une image dynamique des groupements végétaux.

Le principe est simple: il s'agit de reconnaître des systèmes, constitués d'éléments, les éléments phytosociologiques, qui prennent alors valeur d'éléments systémiques, et de relations réunissant ces éléments, les relations (ou transformations) spatio-temporelles, qui prennent valeur de transformations systémiques (De Foucault, 1984 ; De Foucault, 1993). Selon Decocq (1997b), ces systèmes sont habituellement définis pour des régions naturelles aux caractéristiques géologiques et climatiques globalement uniformes pour la végétation.

La phytosociologie (sigmatiste ou synusiale) permettait jusqu'ici de décrire, d'analyser et de classer les groupements 
végétaux grâce à des études texturales (synfloristique $=$ combinaisons floristicostatistiques), structurelles (caractères architecturaux) et fonctionnelles (synécologie $=$ recherche des agents du déterminisme écologique), ce qui correspond à une démarche «intra »(Gillet et al., 1991; De Foucault, 1993). L'approche systémique va plus loin, en analysant les relations spatiotemporelles qui relient entre eux les groupements végétaux précédemment distingués et en permettant, de cette manière, la distinction de systèmes phytosociologiques : il s'agit d'une démarche «inter»( Piaget et Garcia, 1983).

\section{Concepts fondamentaux de systémique végétale}

Pour donner à l'approche systémique de la végétation une dimension universelle, il est indispensable de formaliser son objet d'étude, à savoir la dynamique des communautés végétales. Cette étape donne lieu à la définition d'un certain nombre de concepts fondamentaux dont les principaux ont été synthétisés par Gillet et al. (1991). Nous insistons cependant sur l'adaptation de certains d'entre eux au milieu forestier en général et tropical en particulier.

\section{Successions spatiales et temporelles}

Selon Decocq (1997b), la végétation possède une structure bi-dimensionnelle : spatiale d'une part et temporelle d'autre part. Si l'on considère la dimension spatiale de la végétation, il faut, en fait, distinguer son agencement horizontal, qui consiste en une juxtaposition de groupements végétaux, liée à l'hétérogénéité des conditions écologiques, de son agencement vertical, qui se traduit par une superposition de communautés végétales distinctes liées à la diversité des comportements éthologiques. Toutes ces communautés végétales sont donc structurellement liées et chacune d'elles est reliée aux autres par une relation spatiale, bilatérale dans le cas d'une juxtaposition, unilatérale (dépendance écologique) dans le cas d'une superposition. Dans le premier cas, la relation est interne à la strate végétale considérée (relation spatiale «intrastrate»), tandis que dans le second, la relation intéresse deux strates distinctes d'une même phytocœnose (relation spatiale interstrate).

Par exemple, le long d'un profil topographique, on peut observer différentes communautés végétales en fonction du degré d'hydromorphie du sol; au point le plus bas se trouve un groupement hygrophile et au fur et à mesure que l'on remonte le profil, des communautés de moins en moins hygrophiles se succèdent jusqu'à l'émergence d'une communauté mésophile, voire xérophile. On a donc une succession spatiale de communautés, le passage de l'une à la suivante se faisant grâce à une relation systémique : l'assèchement du sol. Réciproquement, si l'on suit le profil en sens inverse, on passe à des communautés de plus en plus hygrophiles, la relation systémique étant cette fois l'humidification du sol. Chaque communauté correspond à un stade de succession spatiale. La limite entre deux communautés n'est évidemment pas nette : il existe une «zone de contact» plus ou moins large, pour laquelle il est souvent difficile de faire une typologie phytosociologique du fait de sa texture apparemment hétérogène; en effet, s'y côtoient généralement des espèces relictuelles du niveau précédent et des espèces introgressives du niveau suivant (introgression dynamique). Ces zones de contacts ou écotones possèdent donc une réelle signification dynamique et l'approche systémique permet, outre de préciser leur déterminisme, de les intégrer dans les systèmes phytosociologiques.

\section{Notion de système phytosociologique}

Le système phytosociologique, objet d'étude de la systémique végétale, est un ensemble organisé au sein duquel sont rassemblés tous les syntaxons phytosociologiques élémentaires reconnus dans une aire d'étude déterminée (en général, une région naturelle aux conditions climatiques et écologiques globalement uniformes), ainsi que toutes les relations (ou 
transformations) spatiales et temporelles qui les relient entre eux. Les syntaxons phytosociologiques élémentaires deviennent des éléments systémiques (si leur déterminisme écologique est connu) et les relations spatiales et temporelles, des transformations systémiques.

Selon le principe du «parallélisme entre le degré de complexité des objets concrets étudiés et le niveau d'intégration des unités abstraites correspondantes», il est possible de définir plusieurs types de systèmes phytosociologiques, plus ou moins complexes: les systèmes unistrates, les systèmes phytocœnotiques, les systèmes téselaires et les systèmes caténaires (Gillet et al., 1991).

L'intérêt essentiel du concept de système phytosociologique réside dans le fait que les éléments systémiques sont propres à une région naturelle donnée et que les transformations systémiques sont en revanche universelles. Il ouvre ainsi la voie d'un véritable structuralisme de la végétation, valable à l'échelle planétaire (de Foucault, 1984; Decocq, 1999).

\section{Essai de classification des transformations systémiques}

Pour Decocq (1999), l'approche systémique est intéressante du fait que les transformations mises en jeu sont universelles et, finalement, pas très nombreuses. Il est utile dès lors de donner une classification de ces transformations élémentaires, afin de mieux comprendre leur importance. Une première typologie a été proposée par De Foucault (1988), qui distinguait les transformations d'effets négatifs, de celles d'effets positifs variables. Cette conception était assez ambiguë, dans la mesure où presque toutes les transformations peuvent avoir, soit des effets positifs, soit des effets négatifs. La notion même d'effets positifs/ effets négatifs est très relative ; le point de vue du conservateur de la biodiversité n'est certainement pas le même que celui de l'agriculteur.

Decocq (1997b) a montré, qu'il était possible de décomposer tout phénomène dynamique en un certain nombre de transformations élémentaires, caractérisées par leur nature, elle-même quasiment indissociable de leur origine. Pour Gillet et al. (1991), dans l'établissement initial des objets et concepts de la phytosociologie synusiale, un certain nombre de «flèches systémiques » étaient présentées sous forme de tableau, sans réelle classification, mais avec une opposition entre relations temporelles et relations spatiales. Pour Decocq (1997a), il ne devrait pas avoir d'opposition dans une telle classification, c'est pourquoi il a proposé un nouvel essai de classification, fondé sur le principe que tout facteur écologique du milieu est susceptible de subir des variations, indépendamment des autres. Nous ne nous étendrons pas ici sur cet essai de classification de Decocq (1997a). Mais nous présentons simplement le tableau récapitulant les principales transformations spatio-temporelles élémentaires susceptibles d'être utilisées en systémique forestière (Tableau 1).

\section{Représentation des phénomènes phytodynamiques \\ Les éléments systémiques et les} transformations qui les relient étant connus, il devient possible de les intégrer dans un système phytosociologique. Plus celui-ci intégrera d'éléments et plus il possédera une réelle valeur pour d'éventuelles applications ultérieures. Selon Decocq (1997b), l'idéal serait d'obtenir au sein de ces systèmes phytosociologiques des séries phytodynamiques pour lesquelles une seule transformation élémentaire interviendrait, les éléments se succédant alors le long d'un gradient écologique donné. Ces éléments possèdent un certain nombre de caractères communs, invariants dans leur déterminisme causal. Néanmoins, cet auteur ajoute qu'en pratique, cela est souvent beaucoup plus difficile puisque plusieurs facteurs écologiques varient souvent simultanément; il faut alors tenter de déterminer celui qui exerce la plus forte influence sur la végétation. Par exemple l'oligotrophisation d'un sol s'accompagne quasi systématiquement d'une 
N. J. KASSI et al. / Int. J. Biol. Chem. Sci. 5(3): 1337-1350, 2011

Tableau 1 : Essai de classification des transformations spatio-temporelles élémentaires en fonction de leur nature (Decocq, 1997b).

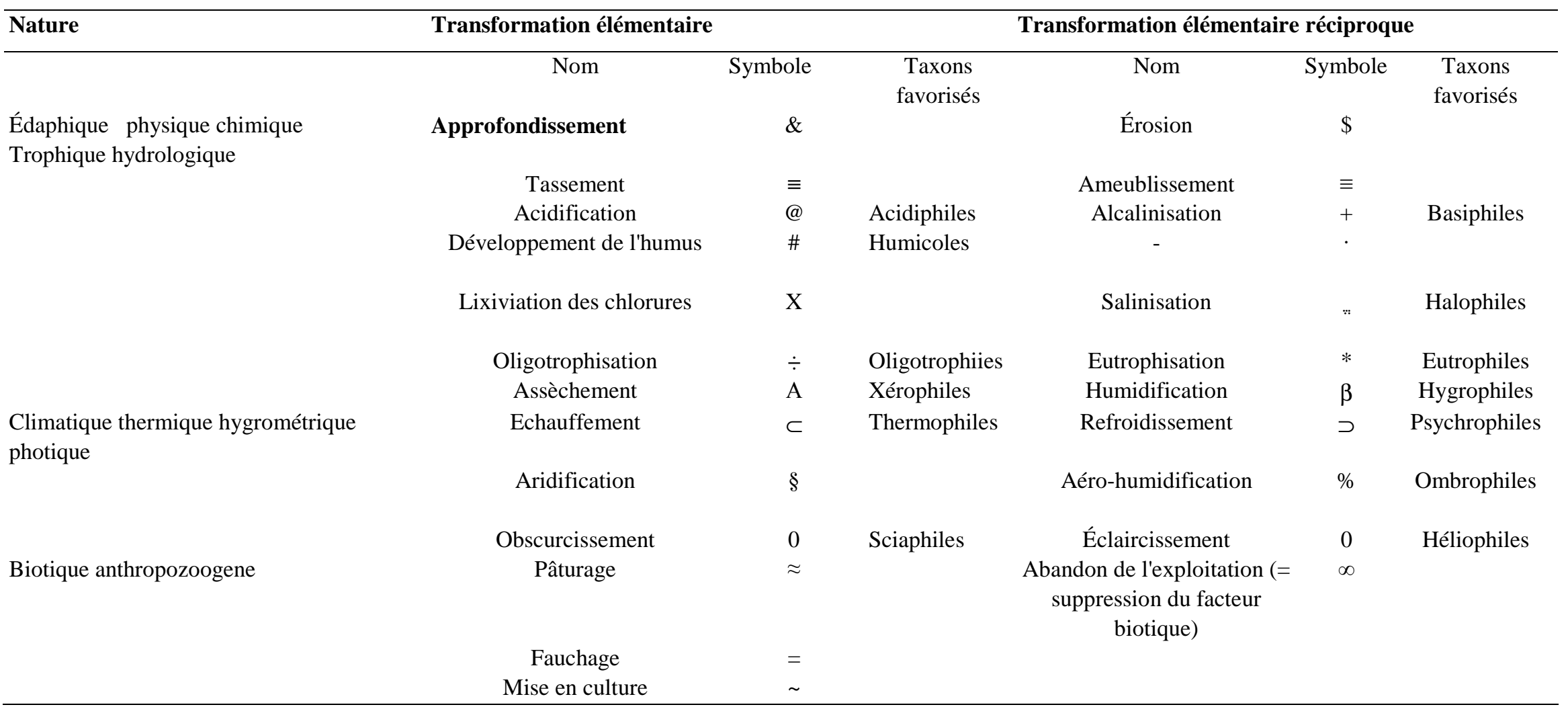


acidification de celui-ci, d'où d'ailleurs la fréquente confusion entre espèces oligotrophiques et espèces acidiphiles.

Une première représentation d'un phénomène phytodynamique peut mettre en avant l'évolution de la synfloristique des différents syntaxons selon un gradient écologique donné, au moyen par exemple d'un «tableau en escalier ». Celui-ci consiste à ordonner selon le gradient écologique (par exemple du syntaxon le plus oligotrophique au syntaxon le plus eutrophique). Les taxons végétaux doivent eux aussi être ordonnés de manière à faire apparaître les liens synfloristiques reliant les syntaxons entre eux, d'où la forme en «escalier» du tableau. A l'intersection entre lignes et colonnes se trouvent les classes de présence des taxons au sein de chaque syntaxon.

Selon Decocq (1999), le tableau «en escalier» permet de décrire une série dynamique sur un plan synfloristique, mais il est également possible de schématiser cette série au moyen d'un graphe systémique. Les éléments systémiques (syntaxons élémentaires en général) sont alors représentés par leur étiquette nomenclaturale et les transformations qui les relient par des flèches symboliques. Les symboles utilisés sont ceux déjà cités précédemment (Tableau 1), chaque transformation élémentaire possédant son symbole; les flèches possèdent une pointe pleine si la transformation considérée est spatiale ou une pointe vide si la transformation considérée est temporelle, sachant que selon le principe de l'isomorphisme spatio-temporel, bon nombre de ces transformations sont interchangeables (De Foucault, 1997b). Toutefois, nous rappelons que ce principe doit être utilisé avec beaucoup de précaution tant que les limites n'en seront pas fixées.

Exemple : Application aux forêts denses semi décidues dans le Centre-Est (Bongouanou Dimbokro ; Côte d'Ivoire)

Pour discuter de ce que pourrait apporter l'approche systémique en matière de foresterie tropicale, nous proposons d'appliquer cette démarche à la végétation forestière (forêt classée de Sanaimbo, CentreEst de la Côte d'Ivoire : $6^{\circ} 20^{\prime}-6^{\circ} 26^{\prime}$ N, 4 $\circ 33^{\prime}-4^{\circ} 37^{\prime} \mathrm{O}$ ), et plus particulièrement aux forêts denses semi-décidues. Dans cette étude, nous avons analysé la végétation de jachères incluses dans une matrice de forêt dense semidécidue, comparativement à celle de forêts anciennes intactes (forêts «primaires ») ou seulement exploitées pour leurs bois, de manière à rechercher si les espèces s'assemblaient en communautés. Soixantequatre jachères ont été échantillonnées le long d'une chronoséquence post-culturale de 30 ans, ainsi que des forêts anciennes intactes ou exploitées, sur deux types de sol: ferrallitique et hydromorphe. Conformément à la méthode synusiale intégrée, nous avons construit une matrice contenant les syntaxons avec leur pourcentage de recouvrement au sol au sein de 80 relevés de phytocœnose. Une CH (méthode de Ward, distances euclidiennes relatives) suivie d'une analyse des espèces indicatrices (ISA) et de leur ordination par analyse des correspondances détendancées (DCA) ont été réalisées sur cette matrice. Les groupes issus de cette analyse ont été interprétés comme des cœnotaxons et nommés selon les recommandations de Gillet et al. (1991). Une typologie phytosociologique a été conduite selon la méthode synusiale intégrée, ce qui permet ultérieurement une analyse très fine des phénomènes phytodynamiques. Toutes les associations végétales et leurs subdivisions citées ci-après ont été décrites dans le cadre de ces précédents travaux, auxquels nous renvoyons le lecteur pour autorité ainsi que pour les différentes caractéristiques synfloristiques et synécologiques (Kassi, 2006 ; Kassi et Decocq, 2007).

\section{Modélisation systémique}

$\mathrm{La}$ dynamique post-culturale a finalement été reconstituée le long de la chronoséquence étudiée, grâce à une approche systémique (Gillet et al., 1991 ; De Foucault, 
1993), déjà validée dans l'étude des systèmes de recolonisation (Decocq, 1999). Cette méthode de modélisation qualitative permet de représenter schématiquement les relations de dynamique spatiale (superposition/ subordination et juxtaposition) et temporelle (succession) qui relient les différents syntaxons issus de l'analyse phytosociologique au sein d'un même système écologique, ici une forêt dense semi-décidue de Côte d'Ivoire. Ce type de modèle permet, outre d'étudier le fonctionnement d'un système dynamique complexe, mais aussi de procéder ultérieurement à des comparaisons avec d'autres systèmes similaires et à des généralisations, voire des prédictions (De Foucault, 1993).

Le modèle qui a été construit repose sur les résultats de l'analyse synchronique d'une chronoséquence spatiale, l'espace se substituant au temps. Cette technique a déjà fait les preuves de sa fiabilité dans ce type de système dynamique (Adler et Lauenroth, 2003).

\section{Le système de recolonisation post-culturale}

L'étude de la dynamique post-culturale de la végétation a été réalisée de manière synchronique, le long d'une chronoséquence spatiale. La classification hiérarchique $(\mathrm{CH})$ des relevés synusiologiques suivie d'une analyse des espèces indicatrices (ISA) et de leur ordination par analyse des correspondances détendancées (DCA) ont permis de décrire 49 syntaxons, interprétés comme 20 associations végétales. La $\mathrm{CH}$, l'ISA et la DCA des relevés phytocœnotiques ont abouti à la distinction de 12 cœnotaxons interprétés comme 7 cœnoassociations (Kassi, 2006 ; Kassi et Decocq, 2007). L'ensemble de ces résultats a finalement été synthétisé dans un modèle qualitatif de la succession postculturale grâce à une approche systémique, fondée sur le postulat d'isomorphisme spatiotemporel, que nous allons résumer dans les lignes qui vont suivre.
On peut émettre l'hypothèse que cette succession spatiale témoigne de ce qui se produit dans le temps, hypothèse qui ne pourrait être validée que par un suivi diachronique de champs abandonnés au sein d'une matrice forestière. D'après les résultats de notre analyse structurelle et phytosociologique de la végétation, on peut synthétiser l'ensemble et modéliser qualitativement les principales étapes de la succession secondaire post-culturale à l'aide d'un graphe systémique (Figure 1).

La colonisation initiale du champ se fait via la mise en place d'une fruticée, dès les premières années suivant l'abandon. Au bout de la cinquième année, cette strate sous-arbustive, occupée par le Solano - Paullinietum pinnatae Kassi, est déjà très dense et dominée quantitativement par l'espèce invasive Chromolaena odorata (L.) R. King \& H. Robinson (Asteraceae). Entre la cinquième et la dixième année, les premiers arbustes «percent» ces fourrés et assurent la mise en place progressive du Voacango-Rauvolfietum vomitariae kassi, en strate arbustive basse, puis du Newbouldio-Millettietum zechianae Kassi, en strate arbustive haute, qui n'atteindront leur optimum qu'autour de la quinzième année. Simultanément, l'ombrage que ces 2 communautés occasionnent sur la fruticée induit le passage à une strate sousarbustive moins héliophile, l'ErythrococcoClausenetum anisatae Kassi. L'ensemble de ces stades pionniers correspond à différentes variations du Centrosemo-Paullinioconetum pinnatae Kassi, cœnoassociation caractérisant la brousse héliophile pionnière post-culturale. Ces stades initiaux diffèrent radicalement de ceux décrits par Guillaumet et Adjanohoun (1971), qui consistaient en des «recrûs herbeux » à Imperata cylindrica (L.) Raeuschel (Poaceae) ou à Pennisetum purpureum Schumach. (Poaceae). Il est probable que la position d'enclaves à l'intérieur d'une matrice forestière des champs que nous avons étudié explique cette différence. D'une part, cette matrice pourrait 
faire écran à la dispersion des Poacées anémochores précitées depuis l'extérieur de la forêt. D'autre part, la proximité du réservoir d'espèces forestières favoriserait celles-ci dans le processus de recolonisation, puisqu'elles sont probablement plus abondantes, tant dans la pluie de diaspores que dans la banque de graines du sol, et qu'elles peuvent même se régénérer ou se disperser localement par voie végétative.

Entre la quinzième et la vingtième année, les premiers arbres se superposent aux ensembles structurels précédents, pour former l'Afzelio-Albizietum zygiae Kassi, qui contient déjà plusieurs futurs grands arbres de l'ensemble structurel supérieur, dont l'optimum est atteint au-delà de la vingtième année. Les strates sous-jacentes prennent toutes un caractère semi-héliophile, les espèces les plus héliophiles disparaissant au profit des premières sciaphytes forestières. La strate herbacée, qui était jusque-là fragmentaire, commence à se structurer et accueille l'Acrocero-Aneilemetum beniniense Kassi, qui, avec le Clerodendro-Penianthetum palutinervis Kassi, strate sous-arbustive, représentent les premières associations semisciaphiles du sous-bois. Cette forêt basse pionnière correspond au PsydraxoFicoconetum exasperatae.

Au-delà de la vingtième année, la voûte forestière se met progressivement en place, d'abord sous une forme fragmentaire, puis sous une forme associant un couvert arborescent relativement continu aux premières essences décidues «émergentes »: le Pterygoto-Albizietum adianthifoliae Kassi, qui semble atteindre son optimum au-delà de la vingt-cinquième année. La superposition de ce nouvel ensemble structurel, associée à la densification de la strate arborescente dominée, induisent d'importantes modifications floristiques au niveau du sousbois; en particulier, les strates arbustives haute et basse hébergent respectivement le Corynantho-Entadetum rheedei Kassi, et le Combreto-Bridelietum atroviridis Kassi, associations résolument semi-sciaphiles. Cette forêt secondaire coïncide avec le CombretoAlbiziocoenetum adianthifoliae Kassi, qui marque le retour de la forêt dense semidécidue. Au fur et à mesure que la forêt se reconstitue, le sous-bois devient de plus en plus sombre et, probablement, plus humide ; les transformations à type d'obscurcissement et d'aéro-humidification constituent donc les «moteurs» de la succession observée au niveau des strates subordonnées.

A ce stade de la dynamique postculturale, il semble que la survenue d'un épisode de perturbation anthropique, équivalent à l'exploitation extensive du bois, soit capable d'entraîner d'importantes modifications floristiques au niveau des strates arbustive, sous-arbustive et herbacée. Plusieurs groupements végétaux «de dégradation » remplacent les associations précitées; ils sont surtout caractérisés par la co-existence d'espèces rudérales plus ou moins héliophiles avec d'authentiques sciaphytes forestières. Cette forêt au sous-bois perturbé correspond à l'AdenopodioStereospermoconetum acuminatae Kassi, (non représenté sur la Figure 1).

Les plus vieilles jachères étudiées dans ce travail n'avaient que 30 ans. La longueur de notre chronoséquence était donc suffisante pour assister à la remise en place d'une forêt dense semi-décidue pluristratifiée et riche en espèces, bien que non encore saturée cœnologiquement puisque le recouvrement des différentes strates est encore bien en deçà de ce qu'il atteint en forêt «primaire ». Cependant, elle ne permet pas d'objectiver un éventuel retour à la forêt "primaire». Nos résultats viennent donc nuancer ceux de Guillaumet et Adjanohoun (1971) et de Kahn (1982), pour qui la reconstitution de la forêt semi-décidue était rapide, avec une composition floristique climacique très vite atteinte et un stade de «vieille forêt secondaire avec sous-bois primaire» pratiquement escamoté. D'autant plus que, quelles que soient les conditions édaphiques 
dans lesquelles se met en place le CombretoAlbizioconetum adianthifoliae Kassi, sa composition floristique le rapproche de la forêt «primaire» des sols ferrallitiques. Des six ensembles structurels, seules les strates arborescentes dominées $\left(\mathrm{A}_{2}\right)$ et sous-arbustive $\left(\mathrm{S}_{3}\right)$ abritent des formes fragmentaires des associations de la forêt primaire, respectivement le Combreto-Zanthoxyletum leprieuri Kassi, et le Dictyandro-Diospyretum monbuttensis Kassi. Dans la véritable forêt «primaire» dense semi-décidue, représentée par le Dictyandro-Morocoenetum mesozygiae Kassi, l'ensemble structurel supérieur $\left(\mathrm{A}_{1}\right)$ est plus recouvrant, plus riche en espèces et possède la physionomie caractéristique des voûtes forestières tropicales semi-décidues, avec l'association d'émergents décidus aux espèces sempervirentes de la matrice. Les ensembles subordonnés sont occupés par des associations végétales plus riches et plus sciaphiles. Cette forêt «primaire» sur sol ferrallitique est spatialement en liaison dynamique avec la forêt «primaire» sur sol hydromorphe, le Gardenio-Miliciocanetum excelsae Kassi.

Enfin, l'exploitation forestière des forêts primaires représente une perturbation majeure capable d'induire une succession secondaire régressive, se traduisant par un enrichissement généralisé des différents ensembles structurels en espèces héliophiles plus ou moins rudérales. L'élément le plus marquant est d'ordre physionomique, avec l'envahissement par les lianes. Dans cette forêt primaire dégradée du DictyandroMorocœenetum chaetacmeccenetosum Kassi, la strate sous-arbustive est la plus affectée puisque se met en place une association originale, le Chaetacmo-Dracaenetum surculosae Kassi, qui apparaît comme un bon indicateur de perturbation. Il est à noter que c'est la même phytocœnose qui se met en place, quel que soit le niveau d'hydromorphie du sol.

Nos résultats montrent que, si la forêt dense semi-décidue de Côte d'Ivoire est capable de retrouver une structure architecturale très proche de celle de la forêt primaire 30 ans seulement après l'abandon d'un champ inclus dans une matrice forestière, elle ne recouvre ni sa richesse spécifique ni sa composition floristique initiale. A ce stade, deux hypothèses peuvent être formulées : 1 ) Au-delà de ce délai de 30 ans, une phase de maturation de la jeune forêt secondaire va survenir et va probablement durer de plusieurs décennies à quelques siècles et aboutir au rétablissement d'une forêt «primaire », dont les différents ensembles structurels seront cœnologiquement saturés; 2) La forêt secondaire observée dans les jachères de 30 ans montre que la succession post-culturale de la végétation est «déviante » (Decocq, 2000), c'est-à-dire que la forêt primaire ne se rétablira jamais, mais sera remplacée par une forêt secondaire mature, de structure et de richesse comparables, mais de composition floristique différente. Le véritable scénario est probablement situé quelque part entre ces deux hypothèses. Par ailleurs, le fait que nous n'ayons pu objectiver, ne serait-ce qu'une amorce de la reconstitution de la forêt primaire hygrophile dans les jachères sur sol hydromorphe, suggère que cette forêt est très peu résiliente, voire pas du tout, comparativement à la forêt primaire sur terre ferme. La dynamique régressive du GardenioMiliciocoenetum excelsae Kassi, n'est pas différente de celle du DictyandroMoroconetum mesozygiae Kassi. La destruction du premier pourrait donc être irréversible ou, du moins, très lentement réversible, ce qui justifie de recommander des mesures de conservation particulières.

\section{Apports de l'approche systémique en phytosociologie forestière tropicale}

$\mathrm{Au}$ terme de cet essai illustré d'un exemple concret, il est intéressant d'envisager les apports de l'approche systémique des phénomènes phytodynamiques en matière de phytosociologie forestière tropicale et plus généralement en foresterie. 
Selon Decocq (1997b, 1999), sur un
plan fondamental tout d'abord, la formalisation des phénomènes phytodynamiques par la construction de systèmes fonctionnels représentés schématiquement à l'aide d'un graphe systémique, permet de mieux rendre compte de la dimension temporelle de la végétation forestière, encore trop souvent considérée comme stable et immuable dans le temps. La méthodologie, relativement simple à mettre en œuvre, fait appel à des données le plus souvent déjà connues, puisque fournies par les typologies phytosociologiques; il s'agit alors de reconnaître la ou les transformations élémentaires qui relient les communautés végétales préalablement identifiées. De plus, il est possible d'isoler de ces systèmes phytosociologiques certaines séries mettant en jeu une même transformation élémentaire, i.e., qui correspond à un gradient écologique particulier. Une telle série est très riche d'informations si elle est accompagnée d'un tableau synthétique «en escalier», ce qui, selon Decocq (1997a), devrait être systématique. Elle fournit alors de précieux renseignements sur la valeur indicatrice des syntaxons élémentaires et de leurs espèces constitutives, à condition que le déterminisme écologique de ces syntaxons soit connu. Le tableau «en escalier» permet également de déterminer l'amplitude écologique d'une espèce végétale au sein de la région naturelle considérée. Par ailleurs, cette «vision dynamique » des phénomènes facilite considérablement l'approche phytosociologique des interfaces entre communautés végétales : les fameuses «zones de contact» ou «zones de transition» bien souvent volontairement boudées des phytosociologues et pourtant riches d'enseignements.

Le mode de représentation des systèmes phytosociologiques (graphes systémiques) est propice à la mise en évidence, d'une part des isomorphismes, ce qui constitue une première étape pour la classification des F-structures, et d'autre part des phénomènes de convergence des séries, ce qui apporte des arguments supplémentaires pour la détermination du degré de maturité d'une forêt et de sa position par rapport aux climax climatique potentiel.

Sur un plan appliqué, l'approche systémique en foresterie tropicale promet d'être fructueuse en terme d'applications à la gestion des espaces sylvatiques, comme elle l'est pour les forêts tempérées (Decocq, 1997b ; Decocq, 2004). Nous ne citerons ici que quelques exemples jugés démonstratifs.

Pour Decocq (1997b), un premier problème qui se pose au gestionnaire forestier est l'identification des «stations forestières », celle-ci faisant habituellement appel à la notion d'espèces indicatrices ou à celle, plus précise, de groupes sociologiques indicateurs. En ayant recours à une méthodologie relativement simple à mettre en œuvre, l'approche systémique fournit des données précises quant à la réelle valeur indicatrice d'un taxon ou d'un syntaxon dans une région naturelle donnée. Ces données restent purement qualitatives, mais l'intérêt de l'approche systémique en matière de «bioindication» ne se limite pas à la reconnaissance des stations forestières; il concerne également le suivi de la gestion forestière, certaines espèces, ou plus souvent certaines communautés végétales, étant d'excellentes indicatrices de l'évolution de la végétation et à travers elles, du milieu forestier tout entier. La connaissance de ces indicateurs promet d'être utile pour une gestion durable des écosystèmes forestiers tropicaux (Decocq, 1997b).

Un second problème peut se poser dans le cas de forêts fortement artificialisées (conversion en peuplement mono ou paucispécifiques d'espèces locales ou exotiques) dont on souhaite connaître les potentialités forestières et où seules des observations effectuées sur les groupements contigus (e.g: jachères, forêt primaire initiale) sont susceptibles de renseigner sur ces potentialités. La 
reconstitution des systèmes herbacés unistrates par Decocq (1997b) et le système de recolonisation des jachères post-culturales en Côte d'Ivoire (Kassi, 2006 ; Kassi et Decocq, 2007), rendu possible par l'approche synusiale, permet de faire le lien entre les communautés intra-forestières et les communautés péri-forestières et extraforestières, plus fortement influencées par les activités humaines. Dès lors, il devient théoriquement possible de déduire les potentialités forestières d'un milieu en analysant les groupements végétaux herbacés en place, pourvu que le milieu en question ne soit pas trop éloigné, spatialement et temporellement, de la forêt. Les lois systémiques induites par l'analyse des phénomènes phytodynamiques possèdent donc une réelle valeur heuristique et sont directement utilisables dans le domaine du génie forestier (Decocq, 1997b).

Un troisième type de problème concerne les moyens à mettre en œuvre pour la gestion optimale et durable d'une parcelle boisée, c'est-à-dire qui concilierait bien sûr la production de bois et la conservation du patrimoine biologique, mais qui intégrerait également les diverses autres fonctions de la forêt, depuis la protection des sols jusqu'à l'accueil du public. Cette «stratégie » est du ressort du génie phytoécologique, tel qu'il a été défini par De Foucault (1988) et repose sur la connaissance, soit des systèmes phytosociologiques locaux lorsque le forestier agit en domaine connu, soit des F-structures s'il agit en domaine inconnu. On perçoit ainsi toute la dimension prédictive de l'approche systémique, puisque le forestier peut théoriquement connaître les effets d'un mode de gestion ou d'une intervention ponctuelle avant même qu'il ne l'ait entreprise.

Si nous nous montrons volontairement optimistes, il faut cependant reconnaître que certains problèmes restent à résoudre en matière de systémique végétale. Par exemple, la cinétique des transformations mériterait bien souvent d'être précisée, ce qui n'est hélas possible que par l'intermédiaire d'études diachroniques souvent lourdes à mettre en œuvre. La réversibilité de certaines transformations, notamment trophiques, et le cas échéant, la vitesse de reconstitution du groupement végétal initial serait des points intéressant à éclaircir. Nous supposons que ces transformations, pour la plupart «déviantes » (Kassi et Decocq, 2007), sont effectivement réversibles, mais selon une cinétique très lente par rapport à celle à laquelle le groupement de dégradation s'est mis en place. On rejoint ici la notion d'hystérésis. De même, le principe de l'isomorphisme spatio-temporel devra être expérimenté à une plus grande échelle pour évaluer précisément le champ d'application et fixer ainsi les limites des observations synchroniques en systémique végétale (Kassi, 2006). Cet essai a fait appel à des données de la phytosociologie synusiale, données qui sont encore peu nombreuses à l'heure actuelle pour la végétation forestière tropicale. Elle n'est cependant pas une première en Afrique tropicale (Yongo, 2002; Senterre, 2005; Noumon et Ganblo, 2005; Yongo et De Foucault, 2007), mais est encore trop rare. Il est bien sûr possible d'appliquer la même démarche systémique en utilisant les données de la phytosociologie sigmatiste (les éléments devenant les associations forestières multistrates) ou même celles de la typologie des stations forestières (les éléments devenant les types de station, c'est-à-dire les phytogéocœnoses); malheureuse-ment, les résultats obtenus sont bien moins précis et les transformations reliant les éléments sont beaucoup plus complexes. Un des principaux intérêts de l'approche systémique reposant sur la mise en évidence de transformations élémentaires, on comprend dès lors la nécessité de disposer de données typologiques plus précises possibles. 
N. J. KASSI et al. / Int. J. Biol. Chem. Sci. 5(3): 1337-1350, 2011

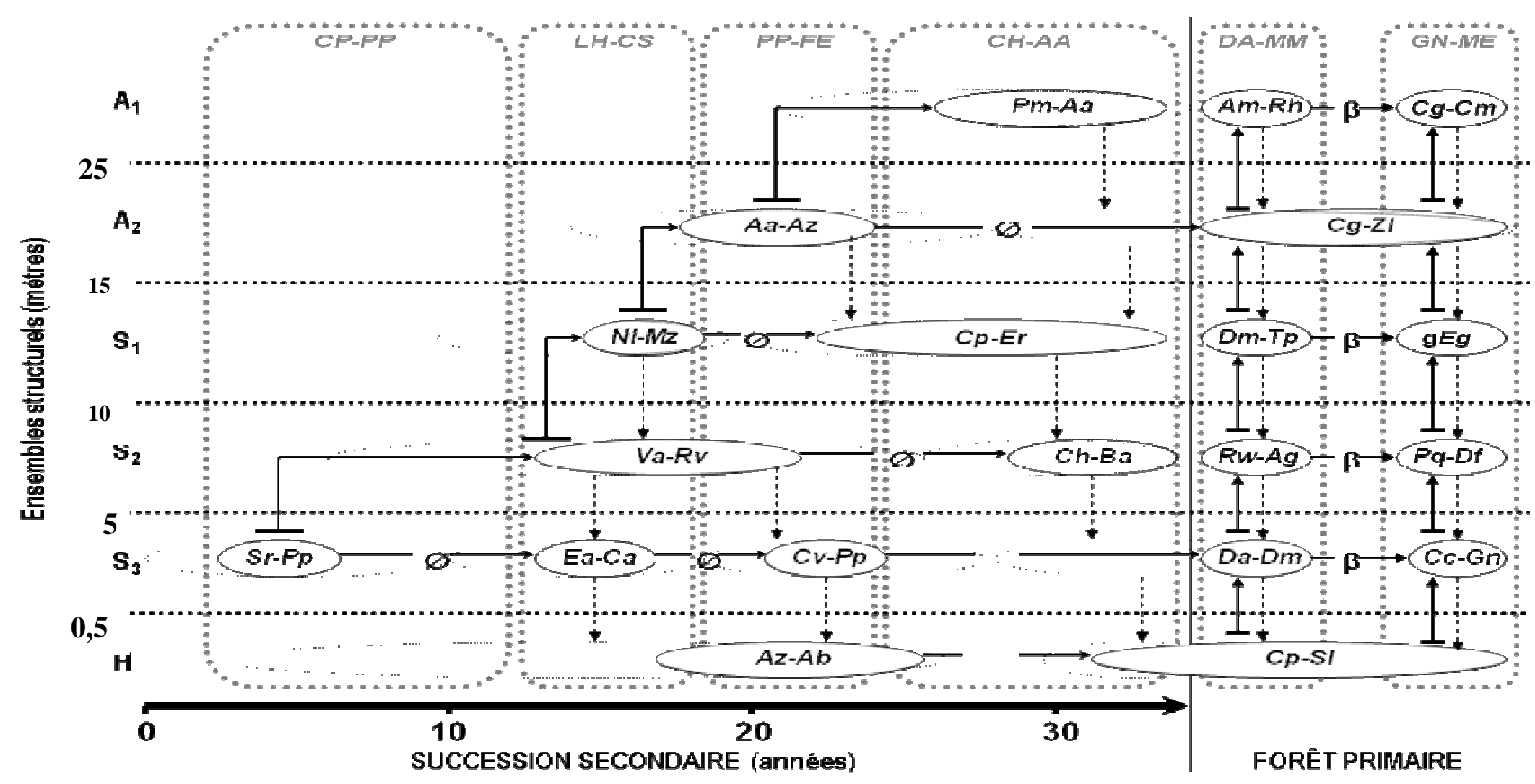

Figure 1 : Modélisation systémique qualitative de la succession forestière post-culturale. : synusies, $:$ : formes fragmentaires,

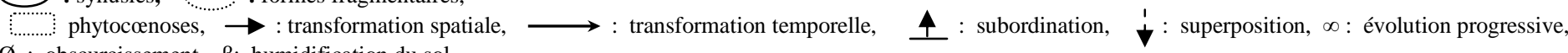
$\varnothing$ : obscurcissement, $\beta$ : humidification du sol. 
Légende de la Figure 1

Conoassociations

CP-PP : Centrosemo pubescentis -

Paullinioconetum pinnatae

LH-CS : Landolphio hirsutae -

Cordioconetum senegalensis

PP-FE : Psydraxo parviflorae - Ficoconetum exasperatae

CH-AA: Combreto hispidi - Albizioconetum adianthifoliae

DA-MM: Dictyandro arborescentis-

Morocœenetum mesozygiae

GN-ME: Gardenio nitidae-Milicioconetum excelsae

Associations synusiales

$\mathrm{S}_{\mathbf{3}}$ : Strate sous-arbustive (de 0 à 5 mètres)

Sr-Pp: Solano rugosi - Paullinietum pinnatae

Ea-Ca: Eryrthrococca anomalae -

Clausenetum anisatae

$\mathrm{Cv}-\mathrm{Pp}$ : Clerodendro volubilis - Penianthetum palutininervis

Da-Dm: Dictyandro arborescentis -

Diospyretum monbuttensis

Cp-Gn: Crotonogyno chevalierii -

Gardenietum nitidae

$\mathrm{S}_{\mathbf{2}}$ : Strate arbustive basse (de 5 à 10 mètres)

Va-Rv: Voacango africanae - Rauvolfietum vomitoriae

Ch-Ba: Combreto hispidi - Bridelietum atroviridis

Rw-Ag: Rinoreo welwitschi - Aidietum genipiflorae

Pq-Df: Premno quadrifoliae - Drypetetum floribundae

$S_{1}$ : Strate arborescente haute (de 10 à 15 mètres)

Ni-Mz: Newbouldio laevis - Millettietum zechianae

Cp-Er: Corynantho pachyceri- Entadetum rheedei

Dm-Tp: Dichapetalo madagascariensis Trichilietum prieureanae

gEg: groupement à Elaeophorbia grandifolia

$A_{2}$ : Strate arborescente dominée (de 15 à 20 mètres)

Aa-Aa: Afzelio africanae - Albizietum zygiae

Cg-Zl: Combreto grandiflori - Zanthoxyletum leprieuri
$A_{1}$ : Strate arborescente haute (plus de 25 mètres)

Pm-Aa: Pterygoto macrocarpae - Albizietum adianthifoliae

Am-Rh: Anthocaryo micrasteris -

Ricinodendretum heudelotii

Cg- Cm: Colo giganteae -Cynometretum megalophyllae

H: Strate herbacée

$\mathrm{Az}$ - Ab: Acrocero zizanoidis - Aneilemetum beniniensis

$\mathrm{Cp}$ - Sl: Ctenitido protensae - Sansevieretum libericae

\section{REFERENCES}

Adler PB, Lauenroth WK. 2003. The power of time: spatiotemporal scaling of species diversity. Ecol. Letters, 6: 749-756.

Decocq G. 1997a. Contribution à l'étude phytosociologique de l'actuelle Theoracia sylva (Thiérache, Aisne, France). Essai d'analyse systémique des phénomènes phytodynamique. Thèse de Doctorat, Université de Lille 2, p. 442.

Decocq G. 1997b. Apports de l'approche systémique des phénomènes phytodynamiques en phytosociologie forestière. Coll. Phytosociologiques, 27: $768-788$.

Decocq G. 1999. La dynamique de recolonisation végétale des anciennes carrières de craies phosphatée du Nord de la France: analyse phytosociologique et systémique. Belgian J. Botany, 132: 77-94.

Decocq G. 2000. Apports de l'approche systémique des phénomènes phytodynamiques en phytosociologie forestière. In Les Données de la Phytosociologie Sigmatiste: Structure, Gestion, Utilisation, Géhu JM. (ed). Cramer J: Berlin ; 767-788.

Decocq G. 2004. Synchronisme, diachronisme et isomorphisme spatio-temporel: intérêt de l'approche archéophytosociologique en systémique végétale. In La Végétation Postglaciaire du Passé et du Présent: Syngénèse, Synécologie, Synsystématique. Géhu JM. (ed). Cramer J: 
Berlin; 803-817.

Foucault (de) B. 1984. Systémique, structuralisme et synsystémique des praires hygrophiles des plaines atlantiques françaises. Thèse de Doct. Sc., Université de Rouen , p. 675.

Foucault (de) B. 1988. Sur les bases épistémologiques du génie écologique, plus spécialement Phyto-écologique. Coll. Phytosociologiques, 15: 77-91.

Foucault (de) B. 1991. Introduction à une systémique des végétations arbustives. Doc. Phytosociogiques, 13: 63-104.

Foucault (de) B. 1993. Systémique qualitative et structuralisme en phytosociologie. Rev. Int. Systématique, 7: 363-384.

Gillet F, Foucault (de) B, Julve P. 1991. La phytosociologie synusiale intégrée : objets et concepts. Candollea, 46: 315340.

Guillaumet JL, Adjanohoun E. 1971. La végétation de la Côte d'Ivoire. In $L e$ Milieu Naturel de la Côte d'Ivoire. Mém. de l'ORSTOM 50: 157-263.

Hartshorn GS. 1980. Neotropical forest dynamics. Biotropica, 12: 23-30.

Kahn F. 1982. La Reconstitution de la Forêt Fropicale après une Culture Traditionnelle (Sud-Ouest de la Côte d'Ivoire). Mém. de l'ORSTOM 97: 1150.

Kassi NJ. 2006. Successions secondaires postculturales en forêt dense semi-décidue de Sanaimbo (Côte d'Ivoire): nature, structure et organisation fonctionnelle de la végétation. Thèse de Doctorat, Université de Picardi Jules Verne, 312p.

Kassi NJ, Decocq G. 2007. Succession secondaire post-culturale en système forestier tropical semi-décidu de Côte d'Ivoire: approche phytosociologique intégrée et systémique. Phytocoenologia, 37(2): 175-219.

Noumon JC, Ganblo JC. 2005. Phytosociologie appliquée à l'aménagement des forêts : cas du périmètre forestier $\mathrm{du}$ Koto (Département du Zou, Centre-Bénin). Acta Botanica Gallica, 152: 269-280.

Piaget J, Garcia R. 1983. Psychogénèse et Histoire des Sciences. Flammarion: Paris ; $310 \mathrm{p}$.

Senterre B. 2005. Recherches méthodologiques pour la typologie de la végétation et la phytogéographie des forêts denses d'Afrique tropicale. Thèse de Doctorat, Université Libre de Bruxelles, p. 343.

Yongo OD. 2002. Contribution aux études floristique, phytogéographique et phytosociologique de la forêt de N'Gotto (R.C.A.). Thèse Doct., Univ. Lille 2, p.347.

Yongo OD, Foucault (de) B. 2007. Contribution à une caractérisation de la végétation de la forêt de N'Gotto (Rép. Centrafricaine). Acta Botanica Gallica, 154(2): 843-857. 